\title{
Fuzzy Soft Semi Continuous Mapping
}

\author{
A. Ponselva kumari ${ }^{1}$, R.Selvi ${ }^{2}$ \\ ${ }^{1}$ (Dept of Mathematics Anna University,Tuticorin Campus ,Tuticorin Tamil Nadu, India. - 628008 \\ ${ }^{2}$ (Dept of Mathematics,Sri ParasakthiCollege for women,.Courtallam Tamil Nadu India -627 802.)
}

\begin{abstract}
In this paper, we study the notions of fuzzy soft semi continuous mapping and we introduce the concept of fuzzy soft semi continuous mapping along with some examples and theorems.

Keywords: Fuzzy soft set, Fuzzy soft topological space, Fuzzy soft mapping, Fuzzy soft identity mapping, Fuzzy soft semi continuous mapping.
\end{abstract}

\section{Introduction}

Molodtsov[5] initiated the novel concept of soft set as a new mathematical tool for dealing with uncertainties in 1999. Maji et al[3] introduced the concept of Fuzzy Soft Set and some properties regarding fuzzy soft union, intersection, complement of a fuzzy soft set Demorgan Law etc. These results were further revised and improved by Ahmad and Kharal[1]. In 2011, Neog et al.[6] put forward some more propositions regarding fuzzy soft set theory and they introduced the notions of union and intersection of two fuzzy soft sets over two fuzzy soft classes.Tanay et al.[12] introduced the definition of fuzzy soft topology in a subset of the initial universe set while Roy and Samanta[10] gave the definition of fuzzy soft topology over the initial universe set.Kharal and Ahmad [1] have been introduced the concepts of fuzzy soft bijective mapping, fuzzy soft identity mapping and fuzzy soft continuous mapping. BanashreeBora[2] studied some propositions and examples related to fuzzy soft continuous mapping. A.ponselvakumari and R.selvi [8\& 9] have been introduced the concepts of fuzzy soft almost continuous mapping (paper accepted) and fuzzy soft pre continuous mapping (paper accepted). In this paper we studied the notions related to Fuzzy soft semi continuous mapping and some related theorems along with examples have been cited.

\section{Preliminaries}

Throughout our discussion, $U$ would refer to an initial universe, $E$, the set of all parameters for $U$ and $\tilde{P}(U)$, the set of all fuzzy subset of $U$ and also by “ $(U, E)$ ", we mean the universal set $U$ and the parameter set $E$ and $S O\left(\mathfrak{J}^{*}\right)$ means semi open of $\mathfrak{J}^{*}$.

\section{Definition 2.1}

Let $A$ be a subset of a topological space $X$. Then $A$ is said to be semi open if $A \subseteq c l(\operatorname{int}(A))$ and semi closed if $\operatorname{int}(\operatorname{cl}(A)) \subseteq A$.

\section{Definition 2.2}

Let $f: X \rightarrow Y$ be a single valued function from a topological space $X$ to a topological space $Y$ Then $f$ is semi continuous if $f^{-1}(V)$ is open in $X$ for every semi open set $V$ in $Y$.

\section{Definition 2.3}

A fuzzy set $A$ in $U$ is a set of ordered pairs $A=\left\{\left(x, \mu_{A}(x)\right): x \in U\right\}$ where $\mu_{A}(x): U \rightarrow[0,1]=I$ is a mapping and $\mu_{A}(x)($ or $A(x))$ states the grade of belongness of $x$ in A. The family of all fuzzy sets in $U$ is denoted by $I^{U}$.

\section{Definition 2.4}

A pair $(F, A)$ is called a fuzzy soft set over $U$ where $F: A \rightarrow \tilde{P}(\mathrm{U})$ is a mapping from A into $\tilde{P}(U)$

\section{Definition 2.5}

Let $A \subseteq E$. Then the mapping $F_{A}: E \rightarrow \tilde{P}(U)$, defined by $F_{A}(e)=\mu^{e} F_{A}$ (a fuzzy subset of $U$ ) is called fuzzy soft set over $(U, E)$, where $\mu^{e} F_{A}=\overline{0}$ if $e \in E-A$ and $\mu^{e} F_{A} \neq \overline{0}$ if $e \in A$. The set of all fuzzy soft set over $(U, E)$ is denoted by $F S(U, E)$. 


\section{Definition 2.6}

Let $(U, E, \mathfrak{I})$ and $\left(\mathrm{U}^{*}, E^{*}, \mathfrak{I}^{*}\right)$ be fuzzy soft topological spaces. Let $\rho: \mathrm{U} \rightarrow U^{*}$ and $\psi: E \rightarrow E^{*}$ be mappings and $f=(\rho, \psi):(\mathrm{U}, \mathrm{E}) \rightarrow\left(\mathrm{U}^{*}, \mathrm{E}^{*}\right)$ be a fuzzy soft mapping

Then $f=(\rho, \psi)$ is said to be fuzzy soft continuous if the inverse image under $f=(\rho, \psi)$ of any $G_{B} \in\left(\mathfrak{J}^{*}\right)$ is a fuzzy soft set $F_{A} \in \mathfrak{I}$ that is $f^{-1}\left(G_{B}\right) \in \mathfrak{I}$ whenever $G_{B} \in\left(\mathfrak{I}^{*}\right)$

\section{Definition 2.7}

Let $(U, E)$ and $\left(U^{*}, E^{*}\right)$ be classes of fuzzy soft sets over $U$ and $U^{*}$ with attributes from $E$ and $E^{*}$ respectively. Let $\rho: U \rightarrow U^{*}$ and $\psi: E \rightarrow E^{*}$ be mappings. Then a fuzzy soft mapping $f=(\rho, \psi):(\mathrm{U}, \mathrm{E}) \rightarrow\left(\mathrm{U}^{*}, \mathrm{E}^{*}\right)$ would be defined as follows-

For a fuzzy soft set $F_{A}$ in $(U, E), f\left(F_{A}\right)$ is a fuzzy soft set in $\left(U^{*}, E^{*}\right)$ obtained as follows: for $\beta \in \psi(E) \subseteq \mathrm{E}^{*}$ and $y \in E^{*}$,

$f\left(\mathrm{~F}_{A}\right)(\beta)(y)=\left\{\begin{array}{l}\bigcup_{x \in \rho^{-1}(y)}\left(\bigcup_{\alpha \in \psi^{-1}(\beta) \cap A} F_{A}(\alpha)\right)(x), \text { if } \rho^{-1}(\mathrm{y}) \neq \varphi, \psi^{-1}(\beta) \cap A \neq \varphi \\ 0, \text { otherwise }\end{array}\right.$

$f\left(F_{A}\right)$ is called fuzzy soft image of the fuzzy soft set $F_{A}$.

\section{Definition 3.1}

\section{Fuzzy Soft Semi Continuous Mapping}

Let $(U, E, \mathfrak{I})$ and $\left(\mathrm{U}^{*}, E^{*}, \mathfrak{I}^{*}\right)$ be fuzzy soft topological spaces. Let $\rho: \mathrm{U} \rightarrow U^{*}$ and $\psi: E \rightarrow E^{*}$ be mappings and $f=(\rho, \psi):(\mathrm{U}, \mathrm{E}) \rightarrow\left(\mathrm{U}^{*}, \mathrm{E}^{*}\right)$ be a fuzzy soft mapping Then $f=(\rho, \psi)$ is said to be fuzzy soft semi continuous if the inverse image under $f=(\rho, \psi)$ of any $G_{C} \in S O\left(\mathfrak{J}^{*}\right)$ is a fuzzy soft set $F_{A} \in \mathfrak{I}$.

That is $f^{-1}\left(G_{C}\right) \in \mathfrak{I}$ whenever $G_{C} \in S O\left(\mathfrak{I}^{*}\right)$.

\section{Example 3.2}

Let $(U, E, \mathfrak{I})$ and $\left(\mathrm{U}^{*}, E^{*}, \mathfrak{I}^{*}\right)$ be fuzzy soft topological spaces. Let $U=\{a, b, c\}$ and $U^{*}=\{x, y, z\} E=\left\{e_{1}, e_{2}, e_{3}, e_{4}\right\} . E^{*}=\left\{e_{1}^{\prime}, e_{2}^{\prime}, e_{3}^{\prime}\right\}$ and $(U, E),\left(U^{*}, E^{*}\right)$ classes of fuzzy soft sets. Let $\rho: U \rightarrow U^{*}$ and $\psi: E \rightarrow E^{*}$ be mappings defined as $\rho(a)=x, \rho(b)=z, \rho(c)=y$ and $\psi\left(e_{1}\right)=e_{1}^{\prime}, \psi\left(e_{2}\right)=e_{3}^{\prime}, \psi\left(e_{3}\right)=e_{2}^{\prime}, \psi\left(e_{4}\right)=e_{1}^{\prime}$.

Let $A_{1}=\left\{e_{1}, e_{2}\right\}, A_{2}=\left\{e_{1}, e_{2}, e_{3}\right\} B_{1}=\left\{e_{1}^{\prime}, e_{2}^{\prime}\right\}, B_{2}=\left\{e_{1}^{\prime}, \mathrm{e}_{2}^{\prime}, e_{3}^{\prime}\right\}$. Let us consider the fuzzy soft sets $\left(F_{A_{3}}, E\right),\left(F_{A_{4}}, E\right)$ and $\left(F_{A_{5}}, E\right)$ in $(U, E)$ and $F_{3}^{3}, F_{4}^{4}, F_{5}^{5}$ in $\left(U^{*}, E^{*}\right)$ as $\left(F_{A_{3}}, E\right)=\left\{\left(e_{1},\{a / 0.5, b / 0.4, c / 0.2\}\right),\left(e_{2},\{a / 0.3, b / 0.5, c / 0.4\}\right),\left(e_{3},\{a / 0.1, b / 0.2, c / 0.3\}\right)\right\}$ $\left(F_{A_{4}}, E\right)=\left\{\left(e_{1},\{a / 0.1, b / 0.1, c / 0.2\}\right),\left(e_{2},\{a / 0.1, b / 0.3, c / 0.1\}\right),\left(e_{3},\{a / 0.3, b / 0.1, c / 0.2\}\right)\right\}$ $\left(F_{A_{5}}, E\right)=\left\{\left(e_{1},\{a / 0.2, b / 0.1, c / 0.3\}\right),\left(e_{2},\{a / 0.1, b / 0.5, c / 0.2\}\right),\left(e_{3},\{a / 0.4, b / 0.3, c / 0.5\}\right)\right\}$ $F_{3}^{3}=\left\{\left(e_{1}^{\prime},\{x / 0.2, y / 0.3, z / 0.1\}\right),\left(e_{2}^{\prime},\{x / 0.4, y / 0.5, z / 0.3\}\right),\left(e_{3}^{\prime},\{x / 0.1, y / 0.2, z / 0.5\}\right)\right\}$ $F_{4}^{4}=\left\{\left(e_{1}^{\prime},\{x / 0.5, y / 0.2, z / 0.4\}\right),\left(e_{2}^{\prime},\{x / 0.1, y / 0.3, z / 0.2\}\right),\left(e_{3}^{\prime},\{x / 0.3, y / 0.4, z / 0.5\}\right)\right\}$ $F_{5}^{5}=\left\{\left(e_{1}^{\prime},\{x / 0.1, y / 0.2, z / 0.1\}\right),\left(e_{2}^{\prime},\{x / 0.3, y / 0.2, z / 0.1\}\right),\left(e_{3}^{\prime},\{x / 0.1, y / 0.1, z / 0.3\}\right)\right\}$ Let

$$
\mathfrak{I}=\left\{\tilde{\varphi}, \tilde{E},\left(F_{A_{3}}, E\right),\left(F_{A_{4}}, E\right),\left(F_{A_{5}}, E\right)\right\} \text { and } \mathfrak{I}^{*}=\left\{\tilde{\varphi}^{*}, \tilde{E}^{*}, F_{3}^{3}, F_{4}^{4}, F_{5}^{5}\right\}, S O\left(\mathfrak{J}^{*}\right)=\left\{\tilde{\varphi}^{*}, \tilde{E}^{*}, F_{4}^{4}, F_{5}^{5}\right\}
$$


where $\tilde{\varphi}^{*}=\left\{\left(e_{1}^{\prime},\{x / 0, \mathrm{y} / 0, \mathrm{z} / 0\}\right),\left(\mathrm{e}_{2}^{\prime},\{x / 0, y / 0, z / 0\}\right),\left(\mathrm{e}_{3}^{\prime},\{\mathrm{x} / 0, \mathrm{y} / 0, \mathrm{z} / 0\}\right)\right\}$

$\tilde{E}^{*}=\left\{\left(\mathrm{e}_{1}^{\prime},\{x / 1, y / 1, z / 1\}\right),\left(e_{2}^{\prime},\{x / 1, y / 1, z / 1\}\right)\left(e_{3}^{\prime},\{x / 1, y / 1, z / 1\}\right)\right\}$

$\tilde{\varphi}=\left\{\left(e_{1},\{\mathrm{a} / 0, \mathrm{~b} / 0, \mathrm{c} / 0\}\right),\left(e_{2},\{a / 0, b / 0, c / 0\}\right)\left(e_{3},\{a / 0, b / 0, c / 0\}\right)\right\} . \tilde{E}=\left\{\left(\mathrm{e}_{1},\{\mathrm{a} / 1, \mathrm{~b} / 1, \mathrm{c} / 1\}\right)\right.$,

$\left.\left(e_{2},\{a / 1, b / 1, c / 1\}\right),\left(e_{3},\{a / 1, b / 1, c / 1\}\right)\right\}$.

Here $f$ is fuzzy soft semi continuous.

\section{Proposition 3.3}

Let i: $\left(U^{*}, E^{*}\right) \rightarrow\left(U^{*}, E^{*}\right)$ be a fuzzy soft identity mapping on a fuzzy soft topological space then $\left(\left(U^{*}, E^{*}, S \mathrm{O}\left(\mathfrak{I}^{*}\right)\right)\right.$ is fuzzy soft semi continuous.

\section{Proof:}

Let $i=(\rho, \psi):\left(U^{*}, E^{*}\right) \rightarrow\left(\mathrm{U}^{*}, \mathrm{E}^{*}\right)$ be the fuzzy soft identity mappings.

Where $\rho: U^{*} \rightarrow \mathrm{U}^{*}$ and $\psi: E^{*} \rightarrow E^{*}$ are identity mappings

Then $\rho(y)=y \quad \forall y \in U^{*}$ and $\quad \psi(\beta)=\beta, \forall \beta \in E^{*}$

$F_{4}^{4} \in S O\left(\mathfrak{I}^{*}\right) \Rightarrow i^{-1}\left(F_{4}^{4}\right)=F_{4}^{4} \in S O\left(\mathfrak{I}^{*}\right)$ and hence $\left(U^{*}, E^{*}, S O\left(\mathfrak{I}^{*}\right)\right)$ is fuzzy soft semi continuous.

\section{Theorem 3.4}

Let $(U, E, \mathfrak{I})$ and $\left(U^{*}, E^{*}, \mathfrak{J}^{*}\right)$ be fuzzy soft topological spaces. Let $\rho: U \rightarrow U^{*}$ and $\psi: E \rightarrow E^{*}$ be mappings and $f=(\rho, \psi):(U, E) \rightarrow\left(U^{*}, E^{*}\right)$ be a fuzzy soft mapping

Then the following statements are equivalent:

(i) The fuzzy soft function $f=(\rho, \psi):(U, E) \rightarrow\left(U^{*}, E^{*}\right)$ is semi continuous.

(ii) The inverse of every fuzzy soft semi closed set is closed.

(iii) For each fuzzy point $e\left(F_{A}\right)$ in $(U, E)$ the fuzzy soft inverse of every neighbourhood $f\left(e\left(F_{A}\right)\right)$ under $f$ is a neighbourhood of $e\left(F_{A}\right)$.

(iv) For each fuzzy soft point $e\left(F_{A}\right)$ in $(U, E)$ and each neighbourhood $G_{B}$ of $f\left(e\left(F_{A}\right)\right)$ there is a neighbourhood $H_{C}$ of $e\left(F_{A}\right)$ such that $f\left(H_{C}\right) \subseteq G_{B}$.

(v) $\left.\quad f\left(\bar{F}_{A}\right) \subseteq \overline{f\left(F_{A}\right.}\right)$.

Proof:

(i) $\Rightarrow$ (ii)

Let $f=(\rho, \psi):(U, E) \rightarrow\left(U^{*}, E^{*}\right)$ be semi continuous and $G_{C} \in S O\left(\mathfrak{I}^{*}\right)$, then $f^{-1}\left(G_{C}\right) \in \mathfrak{I}$.

$G_{C} \in S O\left(\mathfrak{I}^{*}\right) \Rightarrow G_{C}^{C}$ is closed in $\left(U^{*}, E^{*}, \mathfrak{J}^{*}\right)$.

Hence $f^{-1}\left(G_{C}^{C}\right)$ is closed in $(U, E, \mathfrak{I})$.

(ii) $\Rightarrow$ (iii)

Let $H_{B}$ be a neighbourhood of $f\left(e\left(F_{A}\right)\right.$.

Then there is a $G_{C} \in S O\left(\Im^{*}\right)$ such that $f\left(e\left(F_{A}\right)\right) \tilde{\subseteq} G_{c} \tilde{\subseteq} H_{B}$.

That is $e\left(F_{A}\right) \tilde{\in} f^{-1}\left(G_{c}\right) \tilde{\subseteq} f^{-1}\left(H_{B}\right)$,

$f^{-1}\left(G_{C}\right)$ being open in $(U, E, \mathfrak{I})$, the result follows.

(iii) $\Rightarrow$ (iv)

Let $e\left(F_{A}\right) \tilde{\in}(U, E)$ and $G_{C}$ be a neighbourhood of $f\left(e\left(F_{A}\right)\right)$.

Then $f^{-1}\left(G_{C}\right)$ is a neighbourhood of $e\left(F_{A}\right)$.

Thus there exists a fuzzy soft open sets $H_{B}$ in $(U, E)$ such that $e\left(F_{A}\right) \tilde{\in} H_{B} \tilde{\subseteq} f^{-1}\left(G_{C}\right)$ 
$\Rightarrow H_{B} \simeq f^{-1}\left(G_{C}\right)$

$\Rightarrow f\left(H_{B}\right) \tilde{\subseteq} f\left(f^{-1}\left(G_{C}\right)\right) \tilde{\subseteq} G_{C}$

That is $f\left(H_{B}\right) \subseteq G_{C}$.

(iv) $\Rightarrow(v)$

Since $\overline{f\left(F_{A}\right)}$ is fuzzy soft closed in $\left(U^{*}, E^{*}\right), f^{-1} \overline{\left(f\left(F_{A}\right)\right)}$ is fuzzy soft closed in $(U, E)$

Thus $f^{-1} \overline{\left(f\left(F_{A}\right)\right)}=\overline{f^{-1} \overline{\left(f\left(F_{A}\right)\right)}}$

Now $F_{A} \tilde{\subseteq} f^{-1}\left(f\left(\mathrm{~F}_{A}\right)\right) \tilde{\subseteq} f^{-1} \overline{\left(f\left(F_{A}\right)\right)}$, as $f\left(\mathrm{~F}_{A}\right) \tilde{\subseteq} \overline{\left(f\left(F_{A}\right)\right)}$

$\Rightarrow F_{A} \tilde{\subseteq} f^{-1} \overline{\left(f\left(F_{A}\right)\right)}=\overline{f^{-1} \overline{\left(f\left(F_{A}\right)\right)}}$

$\Rightarrow \overline{F_{A}} \subseteq f^{-1}\left(\overline{f\left(F_{A}\right)}\right)$

$\Rightarrow f\left(\overline{F_{A}}\right) \tilde{\subseteq} \overline{f\left(F_{A}\right)}$

$(v) \Rightarrow(\mathrm{i})$

By hypothesis, $f$ is semi continuous.

\section{Example3.5}

Let $(U, E, \mathfrak{I})$ and $\left(\mathrm{U}^{*}, E^{*}, \mathfrak{I}^{*}\right)$ be fuzzy soft topological spaces. Let $U=\{a, b, c\}$ and $U^{*}=\{x, y, z\} E=\left\{e_{1}, e_{2}, e_{3}, e_{4}\right\} . E^{*}=\left\{e_{1}^{\prime}, e_{2}^{\prime}, e_{3}^{\prime}\right\}$ and $(U, E),\left(U^{*}, E^{*}\right)$ classes of fuzzy soft sets. Let $A_{1}=\left\{e_{1}, e_{2}\right\}, A_{2}=\left\{e_{1}, e_{2}, e_{3}\right\} B_{1}=\left\{e_{1}^{\prime}, e_{2}^{\prime}\right\}, B_{2}=\left\{e_{1}^{\prime}, \mathrm{e}_{2}^{\prime}, e_{3}^{\prime}\right\}$

Let us consider the fuzzy soft sets $\left(F_{A_{3}}, E\right),\left(F_{A_{4}}, E\right)$ and $\left(F_{A_{5}}, E\right)$ in $(U, E)$ and $F_{3}^{3}, F_{4}^{4}$ and $F_{5}^{5}$ in $\left(U^{*}, E^{*}\right)$ as

$\left(F_{A_{3}}, E\right)=\left\{\left(e_{1},\{a / 0.5, b / 0.4, c / 0.2\}\right),\left(e_{2},\{a / 0.3, b / 0.5, c / 0.4\}\right),\left(e_{3},\{a / 0.1, b / 0.2, c / 0.3\}\right)\right\}$

$\left(F_{A_{4}}, E\right)=\left\{\left(e_{1},\{a / 0.1, b / 0.1, c / 0.2\}\right),\left(e_{2},\{a / 0.1, b / 0.3, c / 0.1\}\right),\left(e_{3},\{a / 0.3, b / 0.1, c / 0.2\}\right)\right\}$

$\left(F_{A_{5}}, E\right)=\left\{\left(e_{1},\{a / 0.2, b / 0.1, c / 0.3\}\right),\left(e_{2},\{a / 0.1, b / 0.5, c / 0.2\}\right),\left(e_{3},\{a / 0.4, b / 0.3, c / 0.5\}\right)\right\}$

$F_{3}^{3}=\left\{\left(e_{1}^{\prime},\{x / 0.2, y / 0.3, z / 0.1\}\right),\left(e_{2}^{\prime},\{x / 0.4, y / 0.5, z / 0.3\}\right),\left(e_{3}^{\prime},\{x / 0.1, y / 0.2, z / 0.5\}\right)\right\}$

$F_{4}^{4}=\left\{\left(e_{1}^{\prime},\{x / 0.5, y / 0.2, z / 0.4\}\right),\left(e_{2}^{\prime},\{x / 0.1, y / 0.3, z / 0.2\}\right),\left(e_{3}^{\prime},\{x / 0.3, y / 0.4, z / 0.5\}\right)\right\}$

$F_{5}^{5}=\left\{\left(e_{1}^{\prime},\{x / 0.1, y / 0.2, z / 0.1\}\right),\left(e_{2}^{\prime},\{x / 0.3, y / 0.2, z / 0.1\}\right),\left(e_{3}^{\prime},\{x / 0.1, y / 0.1, z / 0.3\}\right)\right\}$

Let

$\mathfrak{I}=\left\{\tilde{\varphi}, \tilde{E},\left(F_{A_{3}}, E\right),\left(F_{A_{4}}, E\right),\left(F_{A_{5}}, E\right)\right\}, \mathfrak{I}^{*}=\left\{\tilde{\varphi}^{*}, \tilde{E}^{*}, F_{3}^{3}, F_{4}^{4}, F_{5}^{5}\right\}$ and $S O\left(\mathfrak{I}^{*}\right)=\left\{\tilde{\varphi}^{*}, \tilde{E}^{*}, F_{4}^{4}, F_{5}^{5}\right\}$

where $\tilde{\varphi}^{*}=\left\{\left(e_{1}^{\prime},\{x / 0, \mathrm{y} / 0, \mathrm{z} / 0\}\right),\left(\mathrm{e}_{2}^{\prime},\{x / 0, y / 0, z / 0\}\right),\left(\mathrm{e}_{3}^{\prime},\{\mathrm{x} / 0, \mathrm{y} / 0, \mathrm{z} / 0\}\right)\right\}$

$\tilde{E}^{*}=\left\{\left(\mathrm{e}_{1}^{\prime},\{x / 1, y / 1, z / 1\}\right),\left(e_{2}^{\prime},\{x / 1, y / 1, z / 1\}\right)\left(e_{3}^{\prime},\{x / 1, y / 1, z / 1\}\right)\right\}$

$\tilde{\varphi}=\left\{\left(e_{1},\{\mathrm{a} / 0, \mathrm{~b} / 0, \mathrm{c} / 0\}\right),\left(e_{2},\{a / 0, b / 0, c / 0\}\right)\left(e_{3},\{a / 0, b / 0, c / 0\}\right)\right\} . \tilde{E}=\left\{\left(\mathrm{e}_{1},\{\mathrm{a} / 1, \mathrm{~b} / 1, \mathrm{c} / 1\}\right)\right.$,

$\left.\left(\mathrm{e}_{2},\{\mathrm{a} / 1, \mathrm{~b} / 1, \mathrm{c} / 1\}\right),\left(\mathrm{e}_{3},\{\mathrm{a} / 1, \mathrm{~b} / 1, \mathrm{c} / 1\}\right)\right\}$.

Let

$e\left(F_{A}\right)=\left(F_{A_{4}}, E\right)=\left\{\left(e_{1},\{a / 0.1, b / 0.1, c / 0.2\}\right),\left(e_{2},\{a / 0.1, b / 0.3, c / 0.1\}\right),\left(e_{3},\{a / 0.3, b / 0.1, c / 0.2\}\right)\right\}$

be a fuzzy soft point in $(U, E)$ and $H_{C}$ be a neighbourhood of $f\left(e\left(F_{A}\right)\right)$, then there is a $F_{5}^{5} \in S O\left(\Im^{*}\right)$ such that $f\left(e\left(F_{A}\right)\right) \simeq F_{5}^{5} \tilde{\subseteq} H_{C}$. 
where

$H_{C}=\left\{\left(e_{1},\{x / 0.4, y / 0.6, z / 0.7\}\right),\left(e_{2},\{x / 0.8, y / 0.6, z / 0.5\}\right),\left(e_{3},\{x / 0.2, y / 0.3, z / 0.4\}\right)\right\}$

$f\left(e\left(F_{A}\right)\right) \in\left(U^{*}, E^{*}\right)$,

$f\left(e\left(F_{A}\right)\right)=F_{5}^{5}=\left\{\left(e_{1}^{\prime},\{x / 0.1, y / 0.2, Z / 0.1\}\right),\left(e_{2}^{\prime},\{x / 0.3, y / 0.2, z / 0.1\}\right),\left(e_{3}^{\prime},\{x / 0.1, y / 0.1, z / 0.3\}\right)\right\}$

Therefore $f\left(e\left(F_{A}\right)\right) \tilde{\subseteq} F_{5}^{5} \simeq H_{C}$.

$\Rightarrow H_{C}$ is a neighbourhood of $f\left(e\left(F_{A}\right)\right)$.

\section{Theorem 3.6}

Let $(U, E, \mathfrak{I})$ and $\left(U^{*}, E^{*}, \mathfrak{I}^{*}\right)$ be fuzzy soft topological spaces. Let $\rho: U \rightarrow U^{*}$ and $\psi: E \rightarrow E^{*}$ be mappings and $f=(\rho, \psi):(U, E) \rightarrow\left(U^{*}, E^{*}\right)$ be a fuzzy soft mapping

Then the following statements are equivalent:

(i) The fuzzy soft function $f=(\rho, \psi):(U, E) \rightarrow\left(U^{*}, E^{*}\right)$ is semi continuous.

(ii) Inverse image of every semi open subset of $\left(U^{*}, E^{*}\right)$ is an open subset of $(U, E)$.

(iii) Inverse image of every semi closed subset of $\left(U^{*}, E^{*}\right)$ is an closed subset of $(U, E)$.

(iv) For each point $e\left(F_{A}\right)$ in $(U, E)$ and for each semi open neighbourhood $F_{5}^{5}$ in $f\left(e\left(F_{A}\right)\right)$, there is a neighbourhood $H_{C}$ of $e\left(F_{A}\right)$ such that $f\left(H_{C}\right) \subset F_{5}^{5}$.

Proof:

(i) $\Rightarrow$ (ii) Let $f=(\rho, \psi):(U, E) \rightarrow\left(U^{*}, E^{*}\right)$ be semi continuous and $G_{C} \in S O\left(\mathfrak{J}^{*}\right)$, then $f^{-1}\left(G_{C}\right) \in \mathfrak{I}$.

Let $F_{5}^{5}$ be any semi open subset of $\left(U^{*}, E^{*}\right)$ and let $e\left(F_{A}\right) \in f^{-1}\left(F_{5}^{5}\right)$. Then $f\left(e\left(F_{A}\right)\right) \in F_{5}^{5}$

Therefore there exists an open set $\left(F_{A_{4}}, E\right)$ in $(U, E)$ such that $e\left(F_{A}\right) \in\left(F_{A_{4}}, E\right)$ and

$f\left(F_{A_{4}}, E\right) \subset{\overline{F_{5}^{5}}}^{0}=F_{5}^{5}$.

Thus $e\left(F_{A}\right) \in\left(F_{A_{4}}, E\right) \subset f^{-1}\left(F_{5}^{5}\right)$ and

Therefore $f^{-1}\left(F_{5}^{5}\right)$ is an neighbourhood of $e\left(F_{A}\right)$.

Hence $f^{-1}\left(F_{5}^{5}\right)$ is open.

(ii) $\Rightarrow$ (iii) Let $E$ be any semi closed subset of $\left(U^{*}, E^{*}\right)$.

Then $\left(U^{*}, E^{*}\right) \sim E$ is semi open and therefore $f^{-1}\left(U^{*}, E^{*}\right) \sim E$ is open.

i.e. $(U, E) \sim f^{-1}(E)$ is open.

Hence $f^{-1}(E)$ is closed.

(iii) $\Rightarrow$ (iv) since $F_{5}^{5}$ is semi open,

$\left(U^{*}, E^{*}\right) \sim F_{5}^{5}$ is semi closed and consequently $f^{-1}\left[\left(U^{*}, E^{*}\right) \sim E\right]$ is closed.

That is $f^{-1}\left(F_{5}^{5}\right)$ is open. Also $e\left(F_{A}\right) \in f^{-1}\left(F_{5}^{5}\right)=H_{C}$.

Then $H_{C}$ is a neighbourhood of $e\left(F_{A}\right)$ such that $f\left(H_{C}\right) \subset F_{5}^{5}$.

(iv) $\rightarrow$ (i)By hypothesis $f$ is semi continuous.

\section{Example 3.7}

Let $(U, E, \mathfrak{I})$ and $\left(\mathrm{U}^{*}, E^{*}, \mathfrak{I}^{*}\right)$ be fuzzy soft topological spaces. Let $U=\{a, b, c\}$ and $U^{*}=\{x, y, z\} E=\left\{e_{1}, e_{2}, e_{3}, e_{4}\right\} . E^{*}=\left\{e_{1}^{\prime}, e_{2}^{\prime}, e_{3}^{\prime}\right\}$ and $(U, E),\left(U^{*}, E^{*}\right)$ classes of fuzzy soft sets. Let 
$\rho: U \rightarrow U^{*}$ and $\psi: E \rightarrow E^{*}$ be mappings defined as $\rho(a)=x, \rho(b)=z, \rho(c)=y$ and $\psi\left(e_{1}\right)=e_{1}^{\prime}$, $\psi\left(e_{2}\right)=e_{3}^{\prime}, \psi\left(e_{3}\right)=e_{2}^{\prime}, \psi\left(e_{4}\right)=e_{1}^{\prime}$.

Let $A_{1}=\left\{e_{1}, e_{2}\right\}, A_{2}=\left\{e_{1}, e_{2}, e_{3}\right\} B_{1}=\left\{e_{1}^{\prime}, e_{2}^{\prime}\right\}, B_{2}=\left\{e_{1}^{\prime}, \mathrm{e}_{2}^{\prime}, e_{3}^{\prime}\right\}$.

Let us consider the fuzzy soft sets $\left(F_{A_{3}}, E\right),\left(F_{A_{4}}, E\right)$ and $\left(F_{A_{5}}, E\right)$ in $(U, E)$ and $F_{3}^{3}, F_{4}^{4}$ and $F_{5}^{5}$ in $\left(U^{*}, E^{*}\right)$ as

$\left(F_{A_{3}}, E\right)=\left\{\left(e_{1},\{a / 0.5, b / 0.4, c / 0.2\}\right),\left(e_{2},\{a / 0.3, b / 0.5, c / 0.4\}\right),\left(e_{3},\{a / 0.1, b / 0.2, c / 0.3\}\right)\right\}$

$\left(F_{A_{4}}, E\right)=\left\{\left(e_{1},\{a / 0.1, b / 0.1, c / 0.2\}\right),\left(e_{2},\{a / 0.1, b / 0.3, c / 0.1\}\right),\left(e_{3},\{a / 0.3, b / 0.1, c / 0.2\}\right)\right\}$

$\left(F_{A_{5}}, E\right)=\left\{\left(e_{1},\{a / 0.2, b / 0.1, c / 0.3\}\right),\left(e_{2},\{a / 0.1, b / 0.5, c / 0.2\}\right),\left(e_{3},\{a / 0.4, b / 0.3, c / 0.5\}\right)\right\}$

$F_{3}^{3}=\left\{\left(e_{1}^{\prime},\{x / 0.2, y / 0.3, z / 0.1\}\right),\left(e_{2}^{\prime},\{x / 0.4, y / 0.5, z / 0.3\}\right),\left(e_{3}^{\prime},\{x / 0.1, y / 0.2, z / 0.5\}\right)\right\}$

$F_{4}^{4}=\left\{\left(e_{1}^{\prime},\{x / 0.5, y / 0.2, z / 0.4\}\right),\left(e_{2}^{\prime},\{x / 0.1, y / 0.3, z / 0.2\}\right),\left(e_{3}^{\prime},\{x / 0.3, y / 0.4, z / 0.5\}\right)\right\}$

$F_{5}^{5}=\left\{\left(e_{1}^{\prime},\{x / 0.1, y / 0.2, z / 0.1\}\right),\left(e_{2}^{\prime},\{x / 0.3, y / 0.2, z / 0.1\}\right),\left(e_{3}^{\prime},\{x / 0.1, y / 0.1, z / 0.3\}\right)\right\}$

Let

$\mathfrak{I}=\left\{\tilde{\varphi}, \tilde{E},\left(F_{A_{3}}, E\right),\left(F_{A_{4}}, E\right),\left(F_{A_{5}}, E\right)\right\}, \mathfrak{I}^{*}=\left\{\tilde{\varphi}^{*}, \tilde{E}^{*}, F_{3}^{3}, F_{4}^{4}, F_{5}^{5}\right\}$ and $S O\left(\mathfrak{I}^{*}\right)=\left\{\tilde{\varphi}^{*}, \tilde{E}^{*}, F_{4}^{4}, F_{5}^{5}\right\}$

where $\tilde{\varphi}^{*}=\left\{\left(e_{1}^{\prime},\{x / 0, \mathrm{y} / 0, \mathrm{z} / 0\}\right),\left(\mathrm{e}_{2}^{\prime},\{x / 0, y / 0, z / 0\}\right),\left(\mathrm{e}_{3}^{\prime},\{\mathrm{x} / 0, \mathrm{y} / 0, \mathrm{z} / 0\}\right)\right\}$

$\tilde{E}^{*}=\left\{\left(\mathrm{e}_{1}^{\prime},\{x / 1, y / 1, z / 1\}\right),\left(e_{2}^{\prime},\{x / 1, y / 1, z / 1\}\right)\left(e_{3}^{\prime},\{x / 1, y / 1, z / 1\}\right)\right\}$

$\tilde{\varphi}=\left\{\left(e_{1},\{\mathrm{a} / 0, \mathrm{~b} / 0, \mathrm{c} / 0\}\right),\left(e_{2},\{a / 0, b / 0, c / 0\}\right)\left(e_{3},\{a / 0, b / 0, c / 0\}\right)\right\} . \tilde{E}=\left\{\left(\mathrm{e}_{1},\{\mathrm{a} / 1, \mathrm{~b} / 1, \mathrm{c} / 1\}\right)\right.$,

$\left.\left(\mathrm{e}_{2},\{\mathrm{a} / 1, \mathrm{~b} / 1, \mathrm{c} / 1\}\right),\left(\mathrm{e}_{3},\{\mathrm{a} / 1, \mathrm{~b} / 1, \mathrm{c} / 1\}\right)\right\}$.

$F_{4}^{4}$ is a subset of $S O\left(\mathfrak{J}^{*}\right)$ and $\left(F_{A_{3}}, E\right)$ is a subset of $\mathfrak{I}$. Then $f^{-1}\left(F_{4}^{4}\right)=\left(F_{A_{3}}, E\right)$.

Here inverse image of a semi open subset is open and hence fis fuzzy soft semi continuous.

\section{Theorem 3.8}

Let $(U, E, \mathfrak{I})$ and $\left(U^{*}, E^{*}, \mathfrak{I}^{*}\right)$ be fuzzy soft topological spaces. Let $\rho: U \rightarrow U^{*}$ and

$\psi: E \rightarrow E^{*}$ be mappings and $f=(\rho, \psi):(U, E) \rightarrow\left(U^{*}, E^{*}\right)$ be a fuzzy soft mapping

If $f$ is fuzzy soft semi continuous then $f$ is fuzzy soft continuous.

Proof:

Let $f$ be fuzzy soft semi continuous and $G_{C} \in S O\left(\mathfrak{I}^{*}\right)$ then $f^{-1}\left(G_{C}\right) \in \mathfrak{I}$.

$G_{C} \in S O\left(\mathfrak{J}^{*}\right) \subseteq \mathfrak{I}^{*}$.

$\Rightarrow G_{C} \in \mathfrak{I}^{*}$.

$\Rightarrow f^{-1}\left(G_{C}\right) \in \mathfrak{I}$.

$\Rightarrow$ inverse image of every open set is open.

Therefore $f$ is fuzzy soft continuous.

\section{Example 3.9}

Let $(U, E, \mathfrak{I})$ and $\left(\mathrm{U}^{*}, E^{*}, \mathfrak{I}^{*}\right)$ be fuzzy soft topological spaces. Let $U=\{a, b, c\}$ and $U^{*}=\{x, y, z\} E=\left\{e_{1}, e_{2}, e_{3}, e_{4}\right\} . E^{*}=\left\{e_{1}^{\prime}, e_{2}^{\prime}, e_{3}^{\prime}\right\}$ and $(U, E),\left(U^{*}, E^{*}\right)$ classes of fuzzy soft sets. Let $\rho: U \rightarrow U^{*}$ and $\psi: E \rightarrow E^{*}$ be mappings defined as $\rho(a)=x, \rho(b)=z, \rho(c)=y$ and $\psi\left(e_{1}\right)=e_{1}^{\prime}$, $\psi\left(e_{2}\right)=e_{3}^{\prime}, \psi\left(e_{3}\right)=e_{2}^{\prime}, \psi\left(e_{4}\right)=e_{1}^{\prime}$.

Let $A_{1}=\left\{e_{1}, e_{2}\right\}, A_{2}=\left\{e_{1}, e_{2}, e_{3}\right\} B_{1}=\left\{e_{1}^{\prime}, e_{2}^{\prime}\right\}, B_{2}=\left\{e_{1}^{\prime}, \mathrm{e}_{2}^{\prime}, e_{3}^{\prime}\right\}$. 
Let us consider the fuzzy soft sets $\left(F_{A_{3}}, E\right),\left(F_{A_{4}}, E\right)$ and $\left(F_{A_{5}}, E\right)$ in $(U, E)$ and $F_{3}^{3}, F_{4}^{4}$ and $F_{5}^{5}$ in $\left(U^{*}, E^{*}\right)$ as

$\left(F_{A_{3}}, E\right)=\left\{\left(e_{1},\{a / 0.5, b / 0.4, c / 0.2\}\right),\left(e_{2},\{a / 0.3, b / 0.5, c / 0.4\}\right),\left(e_{3},\{a / 0.1, b / 0.2, c / 0.3\}\right)\right\}$

$\left(F_{A_{4}}, E\right)=\left\{\left(e_{1},\{a / 0.1, b / 0.1, c / 0.2\}\right),\left(e_{2},\{a / 0.1, b / 0.3, c / 0.1\}\right),\left(e_{3},\{a / 0.3, b / 0.1, c / 0.2\}\right)\right\}$

$\left(F_{A_{5}}, E\right)=\left\{\left(e_{1},\{a / 0.2, b / 0.1, c / 0.3\}\right),\left(e_{2},\{a / 0.1, b / 0.5, c / 0.2\}\right),\left(e_{3},\{a / 0.4, b / 0.3, c / 0.5\}\right)\right\}$

$F_{3}^{3}=\left\{\left(e_{1}^{\prime},\{x / 0.2, y / 0.3, z / 0.1\}\right),\left(e_{2}^{\prime},\{x / 0.4, y / 0.5, z / 0.3\}\right),\left(e_{3}^{\prime},\{x / 0.1, y / 0.2, z / 0.5\}\right)\right\}$

$F_{4}^{4}=\left\{\left(e_{1}^{\prime},\{x / 0.5, y / 0.2, z / 0.4\}\right),\left(e_{2}^{\prime},\{x / 0.1, y / 0.3, z / 0.2\}\right),\left(e_{3}^{\prime},\{x / 0.3, y / 0.4, z / 0.5\}\right)\right\}$

$F_{5}^{5}=\left\{\left(e_{1}^{\prime},\{x / 0.1, y / 0.2, z / 0.1\}\right),\left(e_{2}^{\prime},\{x / 0.3, y / 0.2, z / 0.1\}\right),\left(e_{3}^{\prime},\{x / 0.1, y / 0.1, z / 0.3\}\right)\right\}$

Let

$\mathfrak{I}=\left\{\tilde{\varphi}, \tilde{E},\left(F_{A_{3}}, E\right),\left(F_{A_{4}}, E\right),\left(F_{A_{5}}, E\right)\right\}, \mathfrak{I}^{*}=\left\{\tilde{\varphi}^{*}, \tilde{E}^{*}, F_{3}^{3}, F_{4}^{4}, F_{5}^{5}\right\}$ and $S O\left(\mathfrak{I}^{*}\right)=\left\{\tilde{\varphi}^{*}, \tilde{E}^{*}, F_{4}^{4}, F_{5}^{5}\right\}$

Here $f$ is fuzzy soft semi continuous and fuzzy soft continuous.

Note:

Fuzzy soft continuous does not implies fuzzy soft semi continuous.

\section{Example 3.10}

Let $(U, E, \mathfrak{I})$ and $\left(\mathrm{U}^{*}, E^{*}, \mathfrak{I}^{*}\right)$ be fuzzy soft topological spaces. Let $U=\{a, b, c\}$ and $U^{*}=\{x, y, z\} E=\left\{e_{1}, e_{2}, e_{3}, e_{4}\right\} . E^{*}=\left\{e_{1}^{\prime}, e_{2}^{\prime}, e_{3}^{\prime}\right\}$ and $(U, E),\left(U^{*}, E^{*}\right)$ classes of fuzzy soft sets.

Let $\rho: U \rightarrow U^{*}$ and $\psi: E \rightarrow E^{*}$ be mappings defined as $\rho(a)=x, \rho(b)=z, \rho(c)=y$ and

$\psi\left(e_{1}\right)=e_{1}^{\prime}, \psi\left(e_{2}\right)=e_{3}^{\prime}, \psi\left(e_{3}\right)=e_{2}^{\prime}, \psi\left(e_{4}\right)=e_{1}^{\prime}$.

Let $A_{1}=\left\{e_{1}, e_{2}\right\}, A_{2}=\left\{e_{1}, e_{2}, e_{3}\right\} B_{1}=\left\{e_{1}^{\prime}, e_{2}^{\prime}\right\}, B_{2}=\left\{e_{1}^{\prime}, \mathrm{e}_{2}^{\prime}, e_{3}^{\prime}\right\}$

Let us consider the fuzzy soft sets $\left(F_{A_{6}}, E\right)$ in $(U, E)$ and $F_{6}^{6}$ in $\left(U^{*}, E^{*}\right)$ as

$F_{6}^{6}=\left\{\left(e_{1}^{\prime},\{x / 0.6, y / 0.5, z / 0.4\}\right),\left(e_{2}^{\prime},\{x / 0.3, y / 0.4, z / 0.7\}\right),\left(e_{3}^{\prime},\{x / 0.2, y / 0.5, z / 0.6\}\right)\right\}$

$\left(F_{A_{6}}, E\right)=\left\{\left(e_{1},\{a / 0.6, b / 0.4, c / 0.5\}\right),\left(e_{2},\{a / 0.2, b / 0.6, c / 0.5\}\right),\left(e_{3},\{a / 0.3, b / 0.7, c / 0.4\}\right)\right\}$

Let $\mathfrak{I}=\left\{\tilde{\varphi}, \tilde{E},\left(F_{A_{6}}, E\right)\right\}$, and $\mathfrak{I}^{*}=\left\{\tilde{\varphi}^{*}, \tilde{E}^{*}, F_{6}^{6}\right\}$.

where $\tilde{\varphi}^{*}=\left\{\left(e_{1}^{\prime},\{x / 0, \mathrm{y} / 0, \mathrm{z} / 0\}\right),\left(\mathrm{e}_{2}^{\prime},\{x / 0, y / 0, z / 0\}\right),\left(\mathrm{e}_{3}^{\prime},\{\mathrm{x} / 0, \mathrm{y} / 0, \mathrm{z} / 0\}\right)\right\}$

$\tilde{E}^{*}=\left\{\left(\mathrm{e}_{1}^{\prime},\{x / 1, y / 1, z / 1\}\right),\left(e_{2}^{\prime},\{x / 1, y / 1, z / 1\}\right)\left(e_{3}^{\prime},\{x / 1, y / 1, z / 1\}\right)\right\}$

$\tilde{\varphi}=\left\{\left(e_{1},\{\mathrm{a} / 0, \mathrm{~b} / 0, \mathrm{c} / 0\}\right),\left(e_{2},\{a / 0, b / 0, c / 0\}\right)\left(e_{3},\{a / 0, b / 0, c / 0\}\right)\right\} . \tilde{E}=\left\{\left(\mathrm{e}_{1},\{\mathrm{a} / 1, \mathrm{~b} / 1, \mathrm{c} / 1\}\right)\right.$,

$\left.\left(\mathrm{e}_{2},\{\mathrm{a} / 1, \mathrm{~b} / 1, \mathrm{c} / 1\}\right),\left(\mathrm{e}_{3},\{\mathrm{a} / 1, \mathrm{~b} / 1, \mathrm{c} / 1\}\right)\right\}$.

Here $f$ is fuzzy soft continuous but not fuzzy soft semi continuous.

\section{Theorem 3.11}

Every restriction of an fuzzy soft semi continuous mapping is fuzzy soft semi continuous.

Proof:

Let $f$ be a fuzzy soft semi continuous mapping of $(U, E)$ into $\left(U^{*}, E^{*}\right)$.

Let $\left(F_{A_{3}}, E\right)$ be any subset of $(U, E)$.

For any semi open subset $F_{4}^{4}$ of $\left(U^{*}, E^{*}\right)$.

$\left(f /\left(F_{A_{3}}, E\right)\right)^{-1}\left(F_{4}^{4}\right)=\left(F_{A_{3}}, E\right) \cap f^{-1}\left(F_{4}^{4}\right)$

But $f$ being fuzzy soft semi continuous, and hence $f^{-1}\left(F_{4}^{4}\right)$ is open and

Therefore $\left(F_{A_{3}}, E\right) \cap f^{-1}\left(F_{4}^{4}\right)$ is relatively open subset of $\left(F_{A_{3}}, E\right)$.

That implies $\left(f /\left(F_{A_{3}}, E\right)\right)^{-1}\left(F_{4}^{4}\right)$ is an open subset of $\left(F_{A_{3}}, E\right)$. 
Hence $f /\left(F_{A_{3}}, E\right)$ is fuzzy soft semi continuous.

\section{Example 3.12}

Let $f$ be a fuzzy soft semi continuous.

Let $(U, E, \mathfrak{I})$ and $\left(\mathrm{U}^{*}, E^{*}, \mathfrak{I}^{*}\right)$ be fuzzy soft topological spaces. Let $U=\{a, b, c\}$ and $U^{*}=\{x, y, z\} E=\left\{e_{1}, e_{2}, e_{3}, e_{4}\right\} . E^{*}=\left\{e_{1}^{\prime}, e_{2}^{\prime}, e_{3}^{\prime}\right\}$ and $(U, E),\left(U^{*}, E^{*}\right)$ classes of fuzzy soft sets. Let $\rho: U \rightarrow U^{*}$ and $\psi: E \rightarrow E^{*}$ be mappings defined as $\rho(a)=x, \rho(b)=z, \rho(c)=y$ and $\psi\left(e_{1}\right)=e_{1}^{\prime}$, $\psi\left(e_{2}\right)=e_{3}^{\prime}, \psi\left(e_{3}\right)=e_{2}^{\prime}, \psi\left(e_{4}\right)=e_{1}^{\prime}$.

Let $A_{1}=\left\{e_{1}, e_{2}\right\}, A_{2}=\left\{e_{1}, e_{2}, e_{3}\right\} B_{1}=\left\{e_{1}^{\prime}, e_{2}^{\prime}\right\}, B_{2}=\left\{e_{1}^{\prime}, \mathrm{e}_{2}^{\prime}, e_{3}^{\prime}\right\}$

Let us consider the fuzzy soft sets $\left(F_{A_{3}}, E\right),\left(F_{A_{4}}, E\right)$ and $\left(F_{A_{5}}, E\right)$ in $(U, E)$ and $F_{3}^{3}, F_{4}^{4}$ and $F_{5}^{5}$ in $\left(U^{*}, E^{*}\right)$ as

$\left(F_{A_{3}}, E\right)=\left\{\left(e_{1},\{a / 0.5, b / 0.4, c / 0.2\}\right),\left(e_{2},\{a / 0.3, b / 0.5, c / 0.4\}\right),\left(e_{3},\{a / 0.1, b / 0.2, c / 0.3\}\right)\right\}$

$\left(F_{A_{4}}, E\right)=\left\{\left(e_{1},\{a / 0.1, b / 0.1, c / 0.2\}\right),\left(e_{2},\{a / 0.1, b / 0.3, c / 0.1\}\right),\left(e_{3},\{a / 0.3, b / 0.1, c / 0.2\}\right)\right\}$

$\left(F_{A_{5}}, E\right)=\left\{\left(e_{1},\{a / 0.2, b / 0.1, c / 0.3\}\right),\left(e_{2},\{a / 0.1, b / 0.5, c / 0.2\}\right),\left(e_{3},\{a / 0.4, b / 0.3, c / 0.5\}\right)\right\}$

$F_{3}^{3}=\left\{\left(e_{1}^{\prime},\{x / 0.2, y / 0.3, z / 0.1\}\right),\left(e_{2}^{\prime},\{x / 0.4, y / 0.5, z / 0.3\}\right),\left(e_{3}^{\prime},\{x / 0.1, y / 0.2, z / 0.5\}\right)\right\}$

$F_{4}^{4}=\left\{\left(e_{1}^{\prime},\{x / 0.5, y / 0.2, z / 0.4\}\right),\left(e_{2}^{\prime},\{x / 0.1, y / 0.3, z / 0.2\}\right),\left(e_{3}^{\prime},\{x / 0.3, y / 0.4, z / 0.5\}\right)\right\}$

$F_{5}^{5}=\left\{\left(e_{1}^{\prime},\{x / 0.1, y / 0.2, z / 0.1\}\right),\left(e_{2}^{\prime},\{x / 0.3, y / 0.2, z / 0.1\}\right),\left(e_{3}^{\prime},\{x / 0.1, y / 0.1, z / 0.3\}\right)\right\}$

Let

$$
\mathfrak{I}=\left\{\tilde{\varphi}, \tilde{E},\left(F_{A_{3}}, E\right),\left(F_{A_{4}}, E\right),\left(F_{A_{5}}, E\right)\right\}, \mathfrak{I}^{*}=\left\{\tilde{\varphi}^{*}, \tilde{E}^{*}, F_{3}^{3}, F_{4}^{4}, F_{5}^{5}\right\} \operatorname{and} S O\left(\mathfrak{I}^{*}\right)=\left\{\tilde{\varphi}^{*}, \tilde{E}^{*}, F_{4}^{4}, F_{5}^{5}\right\}
$$

The restriction of function $f$ is $f /\left(F_{A_{3}}, E\right):\left(F_{A_{3}}, E\right) \rightarrow\left(U^{*}, E^{*}\right)$ defined by if the inverse

image under $f /\left(F_{A_{3}}, E\right)$ of any $G_{C} \in S O\left(\mathfrak{I}^{*}\right)$ is a fuzzy soft set $F_{A} \in \mathfrak{I}$,

that is $\left(f /\left(F_{A_{3}}, E\right)\right)^{-1}\left(G_{C}\right) \in \mathfrak{I}$

whenever $G_{C} \in S O\left(\mathfrak{J}^{*}\right)$.

Now $\left(f /\left(F_{A_{3}}, E\right)\right)^{-1}\left(F_{4}^{4}\right)=\left(F_{A_{3}}, E\right)$.

Therefore restriction of $f$ is fuzzy soft semi continuous.

\section{Conclusion:}

Fuzzy soft sets are very popular subject for researchers. In this paper the notions of fuzzy soft semi continuous mapping have been introduced and some related properties and theorems have been established. We have put forward some examples to illustrate our notions.

\section{References}

[1] Ahmad B. and Kharal A, “ On Fuzzy Soft Sets ”, Advances in Fuzzy Systems, Volume 2009,Article ID 586507,pp.1-6.

[2] Banashree Bora, “On Fuzzy Soft Continuous Mapping ”,International Journal for Basic Sciences and Social Sciences (IJBSS) ISSN:2319-2968,Vol.1,Issue 2, August-2012 pp.50-64.

[3] Maji P.K. and Roy A.R., “Soft Set Theory”,Computers and Mathematics with Applications 45, 2003,pp. 555-562.

[4] Maji P.K., Biswas R. and Roy A.R., “Fuzzy Soft Sets ”, Journal of Fuzzy Mathematics, Vol 9,no.3.pp. 2001,589-602,

[5] Molodstov, D.A., "Soft Set Theory - First Re sult”, Computers and Mathematics with Applications, Vol. 37,pp. 1931,1999 .

[6] NeogT.j., Sut D.K., "Theory of Fuzzy Soft Sets from a New Perspective”, International Journal of latest Trends in computing, Vol 2,No 3,September 2011,pp.439-450. 
[7] Neog T.J.,Sut D.K. and Hazarika G.C., “ Fuzzy Soft Topological Spaces ”, International journal of Latest trends in Mathematics,Vol.2, No.1,March 2012,pp.54-67.

[8] PonselvakumariP.,andSelvi R.," Fuzzy Soft Almost Continuous Mapping”, Global Journal of Pure and Applied Mathematics (paper Accepted)

[9] PonselvakumariP.,andSelvi R.," Fuzzy Soft Pre Continuous Mapping”, International journal of Mathematical Science and Applications(paper Accepted)

[10] Roy S. and SamantaT.k., “A Note On Fuzzy Soft Topological Spaces”, Annals of Fuzzy Mathematics and Informatics ,2011.

[11] Singal M.K., and Asha Rani Singal., “ Almost Continuous Mappings”,Yokohama Math.3(1968), 63-73.

[12] Tanay B. and Kandemir M. Burc, "Topological structure of fuzzy soft sets", Computer and Mathematics with Applications 61 (2011)pp.2952-2957.

[13] TugbahanSimsekler.,SaziyeYuksel., “Fuzzy Soft Topological Spaces ”, Annals of Fuzzy Mathematics and Informatics, Vol 5,No.1,(January 2013),pp.87-96. 